\section{Zur Theorie der geometrischen Objekte}

\author{
von S. GozĄB und M. KuCraRzeWskr (Kraków)
}

Die Algebra der geometrischen Objekte in allgemeinen Räumen $X_{n}$ ist bisher, außer der Algebra der Affinoren und Affinordichten und außer ganz besonderen Fällen noch nicht ausgearbeitet. Von algebraischen Kombinationen geometrischer Objekte, die keine Größen darstellen, kann man von vornherein nicht behaupten, daß sie geometrische Objekte sind. Erst die Ableitung der Transformationsregel der Komponenten des Objektes und die Feststellung des Gruppencharakters dieser Regel ist ein Beweis dafür, daß wir es wirklich mit einem geometrischen Objekt zu tun haben.

So z.B., wenn das Objekt der parallelen Übertragung mit den. Komponenten $\Gamma_{\mu \lambda}^{\nu}$ gegeben ist, stellen wir fest, daß die Faltung

$$
T_{\mu} \stackrel{\text { df }}{=} \Gamma_{\mu \lambda}^{\lambda}
$$

ein geometrisches Objekt mit $n$ Komponenten ergibt. Die Transformationsregel der Komponenten dieses Objektes lautet folgendermassen:

$$
\Gamma_{\mu^{\prime}}=\Gamma_{\mu} A_{\mu^{\prime}}^{\mu}-\partial_{\mu^{\prime}} \log |\Delta|
$$

wobei

$$
\text { (3) } \quad \Delta=\left|A_{\nu}^{\nu^{\prime}}\right|, \quad A_{\nu}^{v^{\prime}}=\partial \xi^{\nu^{\prime}} / \partial \xi^{\prime \prime}, \quad A_{\nu^{\prime}}^{\nu}=\partial \xi^{\prime \prime} / \partial \xi^{\nu^{\prime}}, \quad \partial_{\mu}=\partial / \partial \xi^{\prime \prime} \text {. }
$$

Es ist nicht schwer zu beweisen, daß die Regel (2) Gruppencharakter besitzt.

Betrachten wir nun eine beliebige Affinordichte $T_{\mu_{1} \ldots \mu_{q}}^{\lambda_{1} \ldots \lambda_{p}}$ mit dem Transformationsgesetz

$$
T_{\mu_{1}^{\prime} \ldots \lambda_{q}^{\prime}}^{\lambda_{1}^{\prime} \ldots \lambda_{p_{j}^{\prime}}^{\prime}}=\Delta^{r} A_{\lambda_{1}}^{\lambda_{1}^{\prime}} \ldots A_{\lambda_{p}}^{\lambda_{p}^{\prime}} A_{\mu_{1}^{\prime}}^{\mu_{1}^{\prime}} \ldots A_{\mu_{q}^{\prime}}^{\mu_{q}} T_{\mu_{1} \ldots \mu_{q}}^{\lambda_{1} \ldots \lambda_{p}}
$$

und bilden wir das formale Produkt

$$
\Lambda_{\mu_{1} \ldots \mu_{q^{p}}}^{\lambda_{1} \ldots \lambda_{p}} \stackrel{\mathrm{df}}{=} T_{\mu_{1} \ldots \mu_{q}}^{\lambda_{1} \ldots \lambda_{p}} I_{\nu}
$$

Es entsteht die Frage, ob $\Lambda$ ein geometrisches Objekt darstellt. Um diese Frage zu beantworten, berechnen wir die Komponenten von $\Lambda$

im Koordinatensystem $\left(\xi^{p^{\prime}}\right)$. Bedienen wir uns (um die Schreibweise zu kürzen) der Sammelindizes $\left.{ }^{1}\right)$, so können wir schreiben

$$
\begin{aligned}
\Lambda_{b^{\prime} v^{\prime}}^{a^{\prime}} & =T_{b^{\prime}}^{a^{\prime}} \Gamma_{y^{\prime}}=\Delta^{r} T_{b}^{a} A_{a b^{\prime}}^{a^{\prime} b}\left(A_{y^{\prime}}^{y} \Gamma_{p}-\partial_{v^{\prime}} \log |\Delta|\right) \\
& =\Delta^{r} \Lambda_{b v}^{a} A_{a b^{\prime} v^{\prime}}^{a^{\prime} b^{\prime}}-\Delta^{r} T_{b}^{a} A_{a b^{\prime}}^{a^{\prime} b} \partial_{v^{\prime}} \log |\Delta| .
\end{aligned}
$$

Daraus ist zu ersehen, daß in der Transformationsregel für $A$ in expliziter Weise auch die Komponenten des Objektes $T$ auftreten und folglich $\Lambda$ im allgemeinen kein geometrisches Objekt ist. dem

Nehmen wir aber den Spezialfall in Betracht, worin $p=q$ und außer-

$$
T_{\mu_{1} \ldots \mu_{p}}^{\lambda_{1} \ldots \lambda_{p}}=A_{\mu_{1}}^{\lambda_{1}} \ldots A_{\mu_{p}}^{\lambda_{p}}
$$

ist, wobei $A$ den Einheitsaffinor bezeichnet.

$$
\text { In diesem Falle haben wir }
$$

$$
A_{\mu_{1} \ldots \mu_{p}}^{\lambda_{1} \ldots \lambda_{p}}=A_{\mu_{1}}^{\lambda_{1}} \ldots A_{\mu_{p}}^{\lambda_{p}} \Gamma_{v}
$$

und (6) geht in

$$
\Lambda_{b^{\prime} \nu}^{a^{\prime}}=\Lambda_{b v}^{a} A_{a b^{\prime} v^{\prime}}^{a^{\prime} b v}-A_{b^{\prime}}^{a^{\prime}} \partial_{\nu^{\prime}} \log |\Delta|
$$

ïber. Hier stellen wir fest, daß auf der rechten Seite neben der Komponente $\Lambda_{b v}^{a}$ nur die Komponenten der Koordinatentransformation $\left(\xi^{\nu}\right) \rightarrow\left(\xi^{\nu^{\prime}}\right)$ figurieren, so daß das Objekt $\Lambda$ ein geometrisches Objekt darstellen kann. $\mathrm{Da} \beta$ es wirklich ein solches darstellt, folgt daraus, daß (9) Gruppencharakter besitzt. In der Tat haben wir

$$
A_{b^{\prime \prime} v^{\prime \prime}}^{a^{\prime \prime}}=\Lambda_{b^{\prime} v^{\prime}}^{a^{\prime}} A_{a^{\prime} b^{\prime \prime v^{\prime \prime}}}^{a^{\prime \prime} b^{\prime}}-A_{b^{\prime \prime}}^{a^{\prime \prime}} \partial_{\nu^{\prime \prime}} \log \left|\Delta_{1}\right|
$$

wobei $A_{1}=\left|A_{v^{\prime}}^{v^{\prime \prime}}\right|$ ist. Setzen wir nun (9) in (10) ein, so erhalten wir

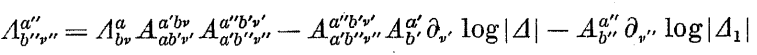

$$
\begin{aligned}
& =A_{b v}^{a} A_{a b^{\prime \prime} v^{\prime \prime}}^{a^{\prime \prime} b v}-A_{b^{\prime \prime}}^{a^{\prime \prime}} \partial_{p^{\prime \prime}} \log \left|\Delta \Delta_{1}\right|, \quad \Delta \Delta_{1}=\left|A_{v}^{p^{\prime \prime}}\right| .
\end{aligned}
$$

Dies beweist, daß (8) ein geometrisches Objekt ist.

Wir gehen jetzt zum Spezialfall $p=1$ über und betrachten das Objekt

$$
A_{\mu \lambda}^{\nu} \stackrel{\mathrm{df}}{=} A_{\lambda}^{v} \Gamma_{\mu} \text {. }
$$

Da das Objekt (12) ein Drei-Indizes-Objekt ist, kann die Frage aufgeworfen werden, ob es vielleicht nicht zur Bestimmung der kovarianten Ableitung für Vektorfelder gebraucht werden kann.

1) J. A. Schouten - D.J. Struik, Einführung in die neueren Methoden der Differentialgeometrie, B. I., S. 32. 
Nehmen wir ein beliebiges Vektorfeld $v^{2}$ und setzen wir

$$
\Phi_{\mu}^{\prime \prime} \stackrel{\text { df }}{=} \partial_{\mu} v^{\nu}+A_{\mu \lambda}^{v} v^{\lambda} \text {. }
$$

Es entsteht die Frage, wann (13) ein Affinorfeld darstellt. Auf Grund der Formeln (9) und (13) haben wir

$$
\begin{aligned}
& \Phi_{\mu^{\prime}}^{v^{\prime}}=\partial_{\mu^{\prime}} v^{v^{\prime}}+\Lambda_{\mu^{\prime} \lambda^{\prime}}^{\prime} v^{\lambda^{\prime}}=\partial_{\mu^{\prime}}\left(A_{\nu}^{v^{\prime}} v^{v^{\prime}}\right)+v^{\lambda^{\prime \prime}}\left(\Lambda_{\mu \lambda}^{\prime \prime} A_{\mu^{\prime} \lambda^{\prime}}^{v^{\prime} \mu \lambda}-A_{\lambda^{\prime}}^{\nu^{\prime}} \partial_{\mu^{\prime}} \log |\Delta|\right) \\
& =A_{\nu \mu^{\prime}}^{\nu^{\prime} \mu} \partial_{\mu} v^{\nu}+v^{\nu} \partial_{\mu} A_{\nu}^{v^{\prime}}+A_{\mu \lambda}^{v} v^{\lambda} A_{\nu^{\prime} \mu^{\prime}}^{v^{\prime} \mu}-v^{v^{\prime}} \partial_{\mu^{\prime}} \log |\Lambda| \\
& =A_{v \mu^{\prime}}^{v^{\prime} \mu} \partial_{\mu} v^{\nu}+A_{\mu^{\prime}}^{\mu} v^{\nu} \partial_{\mu} A_{v^{\prime}}^{v^{\prime}}+\Phi_{\mu}^{\nu} A_{\nu^{\prime}}^{\nu^{\prime} \mu}-A_{\nu^{\prime}}^{\nu^{\prime} \mu} \partial_{\mu} v^{\nu}-A_{v \mu^{\prime}}^{v^{\prime} \prime \prime} v^{\prime \prime} \partial_{\mu} \log |A|,
\end{aligned}
$$

was nach Reduktion

$$
\Phi_{\mu^{\prime}}^{v^{\prime}}=\Phi_{\mu}^{\eta} A_{v^{\prime}}^{\nu^{\prime} \mu}+v^{\prime \prime} A_{\mu^{\prime}}^{\mu}\left(\partial_{\mu} A_{\nu}^{\nu^{\prime}}-A_{\nu}^{v^{\prime}} \partial_{\mu} \log |\Delta|\right)
$$

ergibt. Aus (14) folgt (wenn man berücksichtigt, daß $v$ beliebig wählbar ist), daß (14) dann und nur dann einen Affinor darstellt, wenn die folgenden Relationen

(15) $\quad A_{\mu^{\prime}}^{\mu}\left(\partial_{\mu} A_{v}^{\nu^{\prime}}-A_{v}^{\nu^{\prime}} \partial_{\mu} \log |\Delta|\right)=0 \quad$ für $\nu=1,2, \ldots, n ; \mu^{\prime}, \nu^{\prime}=1^{\prime}, 2^{\prime}, \ldots, n^{\prime}$ erfüllt sind.

Es soll jetzt bewiesen werden, daß für $n>1$ die Beziehungen, (15) folgende Gleichungen

$$
\partial_{\mu} A_{v}^{\nu^{\prime}}=0 \quad\left(\mu, v=1,2, \ldots, n ; v^{\prime}=1^{\prime}, 2^{\prime}, \ldots, n^{\prime}\right)
$$

nach sich ziehen, woraus folgt, daß die Transformation $\left(\xi^{\prime \prime}\right) \rightarrow\left(\xi^{\prime \prime}\right)$ eine lineare ist.

Bei festem $v$ und $v^{\prime}$ stellt (15) ein System von $n$ homogenen Gleichungen mit den Unbekannten $\partial_{\mu} A_{v}^{\nu^{\prime}}-A_{\nu}^{p^{\prime}} \partial_{\mu} \log |\Delta|(\mu=1,2, \ldots, n)$ dar, dessen Determinante $\left|A_{\mu^{\prime}}^{\mu}\right|=\Delta^{-1}$ von Null verschieden ist. Daraus folgt, da $\beta$ (15) dem Gleichungssystem

(17) $\quad \partial_{\mu} A_{v}^{v^{\prime}}-A_{v}^{v^{\prime}} \partial_{\mu} \log |\Delta|=0 \quad\left(\mu, \nu=1,2, \ldots, n ; v^{\prime}=1^{\prime}, 2^{\prime}, \ldots, n^{\prime}\right)$

aequivalent ist. Dividieren wir (17) beiderseits durch $\Lambda$, so nimmt (1.7) folgende Gestalt

$$
\partial_{\mu}\left\{\frac{A_{v}^{p^{\prime}}}{\Delta}\right\}=0
$$

an. Dies besagt, daß die Quotienten $A_{v^{\prime \prime}} / \Delta$ von den $\xi^{\prime \prime}$ unabhängig sind:

$$
A_{\nu}^{v^{\prime}}=\alpha_{\nu}^{\nu^{\prime}} \Delta
$$

wobei also $\alpha_{v}^{v^{\prime}}$ Konstanten sind. Setzen wir in die Determinante $\triangle$ statt $A_{v}^{p^{\prime}}$ die rechten Seiten der Gleichungen (19) ein, so erhält man

$$
\Delta=\left|A_{v}^{\nu^{\prime}}\right|=\left|\Delta \alpha_{\nu}^{\nu^{\prime}}\right|=\Delta^{n}\left|\alpha_{\nu}^{\nu^{\prime}}\right|
$$

woraus $\Delta^{1-n}=\left|\alpha_{v}^{\nu^{\prime}}\right|$ folgt. Für $n>1$ ergibt sich daraus die Beziehung $\Delta=$ const, was zusammen mit (19) zu $A_{y}^{v^{\prime}}=$ const führt, was zu beweisen war.

Wir haben also festgestellt, daß für $n>1$ die Forderung, daß (13) für jedes Vektorfeld $v^{\lambda}$ einen Affinor darstellt, die Einschränkung der allgemeinen (unendlichen) Transformationspseudogruppe der Koordinaten zur (endlichen) affinen Untergruppe zu Folge hat.

Bei der affinen Gruppe stellt schon die gewöhnliche Ableitung $\partial_{\mu} v^{\lambda}$ einen Affinor dar, und man braucht sie nicht mit einem ,Gleichgewichtsglied" ergänzen.

In Spezialfall $n=1$ dagegen sind die Relationen (15) automatisch erfüllt und in diesem Falle - wie bekannt- kann (13) als kovariante Ableitung des Feldes $v^{\lambda}$ erklärt werden. 